\title{
Ma Huang Tang Suppresses the Production and Expression of Inflammatory Chemokines via Downregulating STAT1 Phosphorylation in HaCaT Keratinocytes
}

\author{
Hye-Sun Lim, ${ }^{1}$ Chang-Seob Seo, ${ }^{2}$ Seong-Eun Jin, ${ }^{2}$ Sae-Rom Yoo, ${ }^{2}$ Mee-Young Lee, \\ Hyeun-Kyoo Shin, ${ }^{2}$ and Soo-Jin Jeong ${ }^{1,3}$ \\ ${ }^{1}$ Herbal Medicine Research Division, Korea Institute of Oriental Medicine, Daejeon 34054, Republic of Korea \\ ${ }^{2}$ K-herb Research Center, Korea Institute of Oriental Medicine, Daejeon 34054, Republic of Korea \\ ${ }^{3}$ Korean Medicine Life Science, University of Science \& Technology, Daejeon 34113, Republic of Korea \\ Correspondence should be addressed to Soo-Jin Jeong; sjijeong@kiom.re.kr
}

Received 6 June 2016; Revised 7 September 2016; Accepted 19 September 2016

Academic Editor: Takashi Takeda

Copyright ( $\odot 2016$ Hye-Sun Lim et al. This is an open access article distributed under the Creative Commons Attribution License, which permits unrestricted use, distribution, and reproduction in any medium, provided the original work is properly cited.

Ma huang tang (MHT) is a traditional herbal medicine comprising six medicinal herbs and is used to treat influenza-like illness. However, the effects of MHT on inflammatory skin diseases have not been verified scientifically. We investigated determining the inhibitory effects of MHT against inflammation responses in skin using HaCaT human keratinocyte cells. We found that MHT suppressed production of thymus and activation-regulated chemokine (TARC/CCL17), macrophage-derived chemokine (MDC/CCL22), regulated on activation of normal T-cell expressed and secreted (RANTES/CCL5), and interleukin-8 (IL-8) in tumor necrosis factor- $\alpha$ (TNF- $\alpha$ ) and interferon- $\gamma$ - (IFN- $\gamma$-) stimulated HaCaT cells. Consistently, MHT suppressed the mRNA expression of TARC, MDC, RANTES, and IL- 8 in TNF- $\alpha$ and IFN- $\gamma$-stimulated cells. Additionally, MHT inhibited TNF- $\alpha$ and IFN$\gamma$-stimulated signal transducer and activator of transcription 1 (STAT1) phosphorylation in a dose-dependent manner and nuclear translocation in $\mathrm{HaCaT}$ cells. Our finding indicates that MHT inhibits production and expression of inflammatory chemokines in the stimulated keratinocytes by downregulating STAT1 phosphorylation, suggesting that MHT may be a possible therapeutic agent for inflammatory skin diseases.

\section{Introduction}

Atopic dermatitis $(\mathrm{AD})$ is a frequently occurring inflammatory skin disease associated with severe itching and allergies to environmental factors $[1]$. AD is characterized by the predominant infiltration of macrophages, mast cells, eosinophils, and other inflammatory cells and increased secretion of Th2related response factors by the production of tumor necrosis factor- $\alpha$ (TNF- $\alpha)$ and interferon- $\gamma($ IFN- $-\gamma)$ [2].

Keratinocytes play a pivotal role in the development of inflammatory skin diseases including $\mathrm{AD}$. The cells produced different chemokines and cytokines, especially thymus and activation-regulated chemokine (TARC/CCL17), macrophage-derived chemokine (MDC/CCL22), regulated on activation of normal T-cell expressed and secreted (RANTES/CCL5), and interleukin-8 (IL-8) in response to stimulation by TNF- $\alpha /$ IFN- $\gamma[3,4]$. These mediators are thought to be crucial regulators of the pathogenesis of AD. Additionally, the stimulation of keratinocytes by TNF$\alpha /$ IFN- $\gamma$ leads to the activation of various signaling pathways that involve caspases, mitogen activated protein kinases, nuclear factor-kappa $\mathrm{B}$, and signal transducer and activator of transcription 1 (STAT1) which subsequently increase the expression of inflammatory mediators [5].

Ma huang tang (MHT) comprises six herbal medicines and has traditionally been used in the treatment of sweating, asthma, and febrile disease, such as influenza-like illness (high fever, headache, and cough) [6,7]. Previous research reported that MHT has antipyretic effect in pediatric patients [8]. Other studies showed that MHT has antiasthmatic effects [9]. However, there has been no investigation focusing on the skin inflammatory effects of MHT. Therefore, we investigated 
TABLE 1: Composition of MHT.

\begin{tabular}{|c|c|c|c|c|}
\hline Herbal medicine & Scientific name & Family & Origin & Ratio (\%) \\
\hline Ephedrae Herba & Ephedra sinica Stapf & Ephedraceae & China & 34.9 \\
\hline Cinnamomi Ramulus & Cinnamomum cassia Presl & Lauraceae & Vietnam & 23.3 \\
\hline Glycyrrhizae Radix et Rhizoma & Glycyrrhiza uralensis Fischer & Leguminosae & China & 7.0 \\
\hline Armeniacae Semen & Prunus armeniaca Linne var. ansu Maximowicz & Rosaceae & China & 11.6 \\
\hline Zingiberis Rhizoma Crudus & Zingiber officinale Roscoe & Zingiberaceae & Ulsan, Korea & 11.6 \\
\hline Allii Radix & Allium fistulosum Linne & Liliaceae & Hanam, Korea & 11.6 \\
\hline Total amount & & & & 100.0 \\
\hline
\end{tabular}

the effects and action mechanisms of MHT on the inflammatory mediators using $\mathrm{HaCaT}$ human keratinocytes.

\section{Materials and Methods}

2.1. Plant Materials. MHT consisting of 6 herbs (Table 1), Ephedrae Herba, Cinnamomi Ramulus, Glycyrrhizae Radix et Rhizoma, Armeniacae Semen, Zingiberis Rhizoma Crudus, and Allii Radix, was purchased from Kwangmyungdang Medicinal Herbs (Ulsan, Korea). All raw herbal medicines were confirmed by pharmacognosists, Professor Je-Hyun Lee (College of Oriental Medicine, Dongguk University, Gyeongju, Republic of Korea) and Professor Young-Bae Seo (College of Oriental Medicine, Daejeon University, Gyeongju, Republic of Korea). A voucher specimen (2012KE47-1 KE47-6) has been deposited at the K-herb Research Center, Korea Institute of Oriental Medicine (KIOM).

2.2. Chemicals and Reagents. Cinnamaldehyde ( $\geq 98.0 \%)$, cinnamic acid $(\geq 98.0 \%)$, liquiritin ( $\geq 99.0 \%)$, 6-gingerol $(\geq 98.0 \%)$, and glycyrrhizin $(\geq 99.0 \%)$ were purchased from Wako (Osaka, Japan). Amygdalin and coumarin (both $\geq 99.0 \%$ ) were purchased from Sigma-Aldrich (St. Louis, MO, USA). Liquiritin apioside ( $\geq 98.0 \%)$ was purchased from Shanghai Sunny Biotech (Shanghai, China) and ephedrine $\mathrm{HCl}(\geq 95.0 \%)$ was provided from Ministry of Food and Drug Safety. High-performance liquid chromatography (HPLC) grade, methanol, acetonitrile, and water were purchased from J. T. Baker (Phillipsburg, NJ, USA). Reagent grade, trifluoroacetic acid was purchased from Sigma-Aldrich (St. Louis, MO, USA).

2.3. Preparation of MHT Water Extract. MHT water extract was prepared in our laboratory, KIOM. Namely, the 6 crude herbs, Ephedrae Herba (1,744 g), Cinnamomi Ramulus (1,163 g), Glycyrrhizae Radix et Rhizoma (349 g), Armeniacae Semen (581 g), Zingiberis Rhizoma Crudus (581 g), and Allii Radix (581 g), were mixed and extracted with $50 \mathrm{~L}$ of distilled water at $100^{\circ} \mathrm{C}$ for $2 \mathrm{~h}$ under pressure $(98 \mathrm{kPa})$ using an electric extractor (COSMOS-660; Kyungseo Machine Co., Incheon, Korea). The extracted solution was filtrated through the standard sieve (number 270, $53 \mu \mathrm{m}$; Chung Gye Sang Gong Sa, Seoul, Korea) and then the filtered solution was freeze-dried to obtain a powder using freezing dryer, PVTFD10RS (IlShinBioBase, Yangju, Korea). The amount of freezing-dried MHT powder obtained was $226.2 \mathrm{~g}$ (yield: $4.5 \%)$.

2.4. Chromatographic Analysis of MHT Sample. Chromatographic analysis of the marker components in MHT was performed using the Shimadzu Prominence LC-20A series HPLC system (Kyoto, Japan) equipped with photodiode array (PDA) detector and Lab Solution software (Version 5.54 SP3, Shimadzu, Kyoto, Japan). Waters SunFire $\mathrm{C}_{18}$ analytical column $(250 \times 4.6 \mathrm{~mm} ; 5 \mu \mathrm{m}$, Milford, MA, USA) was used for the separation of the main components as the stationary phase and maintained at $40^{\circ} \mathrm{C}$. The mobile phases consisted of $0.1 \%(\mathrm{v} / \mathrm{v})$ trifluoroacetic acid in distilled water $(\mathrm{A})$ and acetonitrile (B) and the gradient elution for chromatographic separation was as follows: $10-60 \%$ B for $0-30 \mathrm{~min}, 60-100 \%$ B for $30-40 \mathrm{~min}, 100 \% \mathrm{~B}$ for $40-45 \mathrm{~min}, 100-10 \% \mathrm{~B}$ for $45-50 \mathrm{~min}$, and $10 \% \mathrm{~B}$ for $50-60 \mathrm{~min}$. The flow rate was $1.0 \mathrm{~mL} / \mathrm{min}$ and injection volume was $10 \mu \mathrm{L}$.

2.5. Cell Culture. The HaCaT human keratinocyte cell line was obtained from CLS Cell Lines Service GmbH (Eppelheim, Baden-Württemberg, Germany). The HaCaT cells were cultured in Dulbecco's modified Eagle's medium (DMEM, Gibco-BRL, Invitrogen Life Technologies, Inc., Carlsbad, CA, USA) supplemented with $10 \%$ heat-inactivated fetal bovine serum (FBS, Gibco-BRL), penicillin (100 $\mu \mathrm{g} / \mathrm{mL}$; Gibco$\mathrm{BRL})$, and streptomycin (100 $\mu \mathrm{g} / \mathrm{mL}$; Gibco-BRL) in an incubator containing $5 \% \mathrm{CO}_{2}$ at $37^{\circ} \mathrm{C}$.

2.6. Cytotoxicity Assay. Cell viability was assessed using a Cell Counting Kit- 8 assay (CCK- 8 from Dojindo, Kumamoto, Japan) according to the manufacturer's instructions. HaCaT cells $\left(1 \times 10^{3}\right.$ cells/well $)$ were incubated in 96-well plates with various concentrations of the MHT for $24 \mathrm{~h}$. CCK-8 reagent was added to each well and cells were incubated for an additional $4 \mathrm{~h}$. The absorbance was measured at $450 \mathrm{~nm}$ using a Benchmark Plus microplate reader (Bio-Rad Laboratories, Hercules, CA, USA). The percentage of viable cells was calculated as follows: cell viability (\%): (mean absorbance in test well/mean absorbance in control well $) \times 100$.

2.7. Measurement of Chemokine Production. HaCaT cells $(1 \times$ $10^{6}$ cells/well) were cultured in 6-well plates. After reaching confluency, the cells were washed and treated with MHT in $1 \mathrm{~mL}$ of serum-free medium containing TNF- $\alpha$ and IFN- $\gamma$ 
TABLE 2: List of primer sequences for RT-qPCR.

\begin{tabular}{|c|c|c|c|}
\hline \multirow{2}{*}{ Gene } & \multicolumn{2}{|c|}{ Sequences of primers $\left(5^{\prime}\right.$ to $\left.3^{\prime}\right)$} & \multirow{2}{*}{ Size $(b p$} \\
\hline & Forward & Reverse & \\
\hline TARC & ACT GCT CCA GGG ATG CCA TCG TTT TT & ACA AGG GGA TGG GAT CTC ССТ CAC TG & 270 \\
\hline MDC & AGG ACA GAG CAT GGC TCG CCT ACA GA & TAA TGG CAG GGA GCT AGG GCT CCT GA & 362 \\
\hline RANTES & CCC CGT GCC GAG ATC AAG GAG TAT TT & CGT CCA GCC TGG GGA AGG TTT TTG TA & 313 \\
\hline IL-8 & GTG GCT CTC TTG GCA GCC TTC CTG AT & TCT CCA CAA CCC TCT GCA CCC AGT TT & 253 \\
\hline GAPDH & GTG ATG GCA TGG ACT GTG GT & AAG GGT CAT CAT CTC TGC CC & 204 \\
\hline
\end{tabular}

(TI, each $10 \mathrm{ng} / \mathrm{mL}$; R\&D Systems Inc., Minneapolis, MN, USA) for $24 \mathrm{~h}$. The supernatant fractions were harvested, and production of TARC, MDC, RANTES, and IL-8 was determined using a sandwich immunoassay, performed according to the protocols provided by R\&D Systems.

2.8. Reverse Transcription-Polymerase Chain Reaction (RT$P C R)$. Total RNA was isolated using TRIzol reagent according to the manufacturer's instructions (Invitrogen Life Technologies, Inc.). HaCaT cells $\left(1 \times 10^{6}\right.$ cells/well $)$ were cultured to $80-90 \%$ confluency in 6 -well plates. When the cells reached confluence, the cells were washed and treated with MHT in $1 \mathrm{~mL}$ serum-free medium (Gibco-BRL) supplemented with TI for $24 \mathrm{~h}$. Silymarin (Sigma-Aldrich Inc., St. Louis, MO) was used as a positive control drug. Total RNA $(1 \mu \mathrm{g} / \mathrm{mL})$ was then converted into cDNA using an iScript cDNA Synthesis Kit (Bio-Rad Laboratories, Inc.), containing oligo-dT primers. Diethylpyrocarbonate-treated water was added to a final volume $20 \mu \mathrm{L}$ followed by incubation at $42^{\circ} \mathrm{C}$ for $30 \mathrm{~min}$ using a Bio-Rad iCycler apparatus (BioRad Laboratories, Inc.). For PCR amplification, gene-specific primers sequences are listed in the $5^{\prime}$ to $3^{\prime}$ orientation in Table 2 . The PCR reaction mixture contained $1 \mu \mathrm{L} c D N A$ and $1.56 \mu \mathrm{L} \gamma \mathrm{Taq}$ PCR master mix (EBT-1014; Elpis Biotech, Inc., Daejeon, Korea), which contained $1.5 \mathrm{mM} \mathrm{MgCl}_{2}, 0.1 \mu \mathrm{M}$ of each forward and reverse primer and $7.44 \mu \mathrm{L}$ water in a final volume of $10 \mu \mathrm{L}$. The thermocycling program comprised initial denaturation at $94^{\circ} \mathrm{C}$ for $5 \mathrm{~min}$, followed by 25 cycles of denaturation at $94^{\circ} \mathrm{C}$ for $30 \mathrm{sec}$, annealing at $64^{\circ} \mathrm{C}$ for $1 \mathrm{~min}$, extension at $72^{\circ} \mathrm{C}$ for $1 \mathrm{~min} 30 \mathrm{sec}$ for all of the chemokines, and 25 cycles of denaturation at $94^{\circ} \mathrm{C}$ for $30 \mathrm{sec}$, annealing at $52^{\circ} \mathrm{C}$ for $1 \mathrm{~min}$, and extension at $72^{\circ} \mathrm{C}$ for $1 \mathrm{~min} 30 \mathrm{sec}$ for GAPDH. A final extension step was conducted at $72^{\circ} \mathrm{C}$ for $7 \mathrm{~min}$. The amplified products were separated by $1.5 \%$ agarose gel and visualized using Loading STAR staining (A750; DYNE Bio, Seongnam, Korea). The relative expression levels of TARC, MDC, RANTES, and IL-8 mRNA were normalized to those of GAPDH mRNA using a Chemi-Doc Band Analysis system (Bio-Rad Laboratories, Inc.).

2.9. Western Blotting. HaCaT cells were treated with various concentrations of MHT for $1 \mathrm{~h}$ and then incubated in the presence of TI for $30 \mathrm{~min}$. The cells were collected by centrifugation, washed twice with PBS, and suspended in the NEPER Nuclear and Cytoplasmic Extraction Reagents (Thermo Scientific, Rockford, IL) containing protease inhibitors. The protein concentration was determined using a protein assay reagent (Bio-Rad Laboratories, Inc.) according to the manufacturer's instructions. Nuclear protein $(30 \mu \mathrm{g})$ was resolved by $10 \%$ sodium dodecyl sulfate-polyacrylamide gel electrophoresis (SDS-PAGE) and transferred to a polyvinylidene difluoride membrane. The membrane was incubated with blocking solution [5\% skim milk in Tris-buffered saline containing $0.1 \%$ Tween 20 (TBST)], followed by an overnight incubation at $4^{\circ} \mathrm{C}$ with the appropriate primary antibody. The following primary antibodies and dilution were used: antiSTAT1 and antiphospho-STAT1 (1:1000 dilution; Abcam, Cambridge, UK). The membranes were washed three times with TBST and then incubated with a 1:3000 dilution of a horseradish peroxidase- (HRP-) conjugated secondary antibody (Jackson ImmunoResearch, PA) for $1 \mathrm{~h}$ at room temperature. The membranes were again washed three times with TBST and then developed using an enhanced chemiluminescence kit (Thermo scientific, Rockford, IL). Image capture was performed using Chemi-Doc XRS ${ }^{+}$system (BioRad Laboratories, Inc.).

2.10. Immunofluorescence Staining. HaCaT cells were seeded onto glass coverslips and incubated with TI in the absence or presence of MHT $(500 \mu \mathrm{g} / \mathrm{mL})$ for $30 \mathrm{~min}$. The cells were fixed in $4 \%$ paraformaldehyde and $100 \%$ acetone, blocked in $0.5 \%$ bovine serum albumin, and incubated with anti-STAT1 antibody (Cell Signaling, Danvers, MA) for $1 \mathrm{~h}$ at room temperature. Then, fluorescein isothiocyanate-conjugated antirabbit immunoglobulin $\mathrm{G}$ antibody (Invitrogen, Carlsbad, CA, USA) was used as a secondary antibody. The immunostained cells were mounted with medium containing $4^{\prime} 6$ diamidino-2-phenylindole (DAPI) and visualized using an Olympus FLUOVIEW FV10i confocal microscope (Tokyo, Japan).

2.11. Statistical Analysis. The data are expressed as the mean \pm SEM. Data were analyzed using one-way analysis of variance and Dunnett's multiple comparisons test. Results with a $P$ value $<0.05$ were considered to be statistically significant.

\section{Results}

3.1. HPLC Determination of the Nine Marker Components in MHT. The optimized HPLC-PDA analytical method was successfully applied for the simultaneous determination of the 9 marker compounds in MHT extract. All compounds in MHT extract were identified based on the retention time and UV spectra of each reference standard. As a 


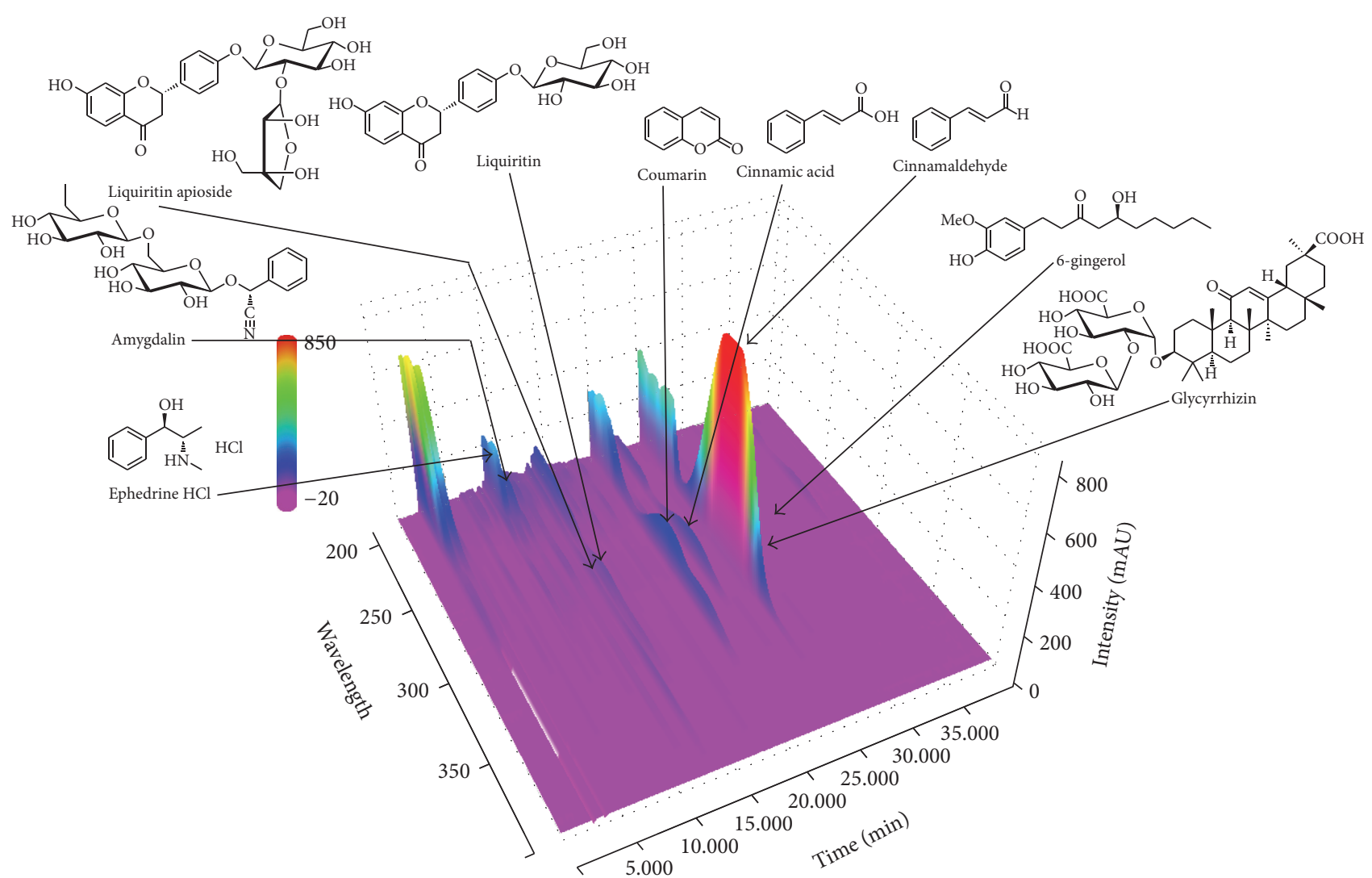

FIGURE 1: Three-dimensional chromatogram of MHT water extract by HPLC-PDA.

result, the 9 marker compounds including ephedrine $\mathrm{HCl}$, amygdalin, liquiritin apioside, liquiritin, coumarin, cinnamic acid, cinnamaldehyde, glycyrrhizin, and 6-gingerol were detected at 9.09, 9.98, 13.89, 14.29, 20.34, 22.80, 25.49, 26.20, and $31.18 \mathrm{~min}$, respectively (Figure 1). The concentrations of ephedrine $\mathrm{HCl}$, amygdalin, liquiritin apioside, liquiritin, coumarin, cinnamic acid, cinnamaldehyde, glycyrrhizin, and 6-gingerol in MHT extract by the optimized analytical assay were $13.80,21.57,4.52,2.08,4.61,1.75,12.52,6.71$, and $0.19 \mathrm{mg} / \mathrm{g}$, respectively.

3.2. Effects of MHT on the Cell Viability in HaCaT Keratinocytes. To obtain a suitable concentration range for investigating the effects of MHT on the viability in HaCaT cells (Figure 2), we treated cells with the concentration ranging from 62.5 to $1000 \mu \mathrm{g} / \mathrm{mL}$ of MHT for $24 \mathrm{~h}$. We observed no significant alteration in the cell viability following MHT treatment up to $1000 \mu \mathrm{g} / \mathrm{mL}$. Therefore, nontoxic concentrations $(125,250$, or $500 \mu \mathrm{g} / \mathrm{mL})$ of MHT were used in subsequent experiments. Additionally, MHT had no effect on cell death by pH level (Supplementary Figure 1 in Supplementary Material available online at http://dx.doi.org/10.1155/2016/ 7831291).

3.3. Effects of MHT on the Inflammatory Chemokines in HaCaT Keratinocytes. To assess the inhibitory effects of MHT, we cotreated HaCaT cells with TI in the absence or presence of MHT for $24 \mathrm{~h}$, and the production of TARC,

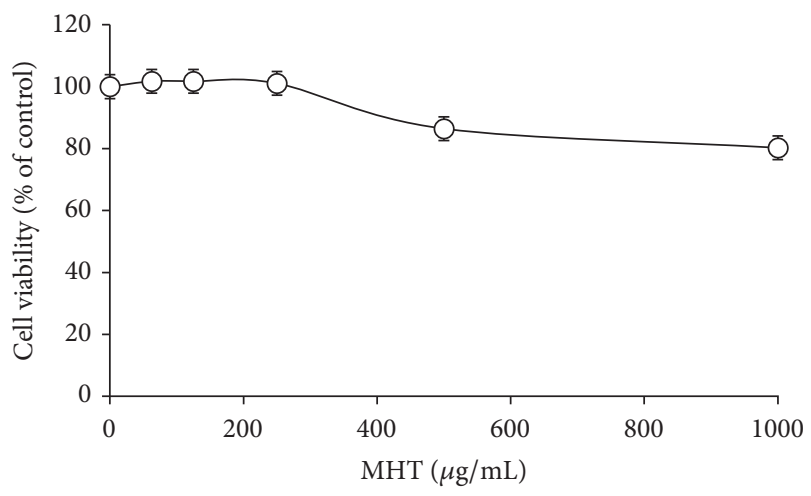

Figure 2: Cytotoxic effects of MHT in HaCaT cells. Cells were seeded onto 96-well plates and treated with various concentrations of $\operatorname{MHT}(0,62.5,125,250,500$, or $1000 \mu \mathrm{g} / \mathrm{mL})$ for $24 \mathrm{~h}$. Cell viability was assessed using CCK- 8 assay. The values are expressed as the mean \pm SEM of three independent experiments.

MDC, RANTES, and IL-8 was analyzed using ELISA. HaCaT cells treated with TI increased the chemokine production compared with the vehicle-treated cells. By contrast, MHT $(125,250$, or $500 \mu \mathrm{g} / \mathrm{mL})$ significantly suppressed TIstimulated production of TARC, MDC, RANTES, and IL8 in a dose-dependent manner $(P<0.01)$, respectively (Figure 3). A positive control silymarin also suppressed the TI-stimulated chemokine production in a dose-dependent 


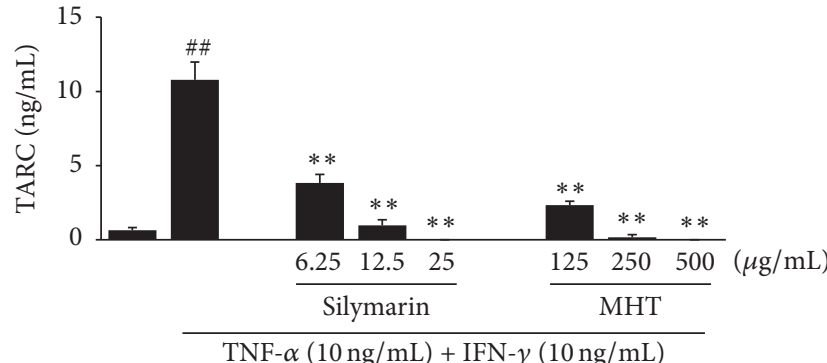

(a)



TNF- $\alpha(10 \mathrm{ng} / \mathrm{mL})+\mathrm{IFN}-\gamma(10 \mathrm{ng} / \mathrm{mL})$

(c)

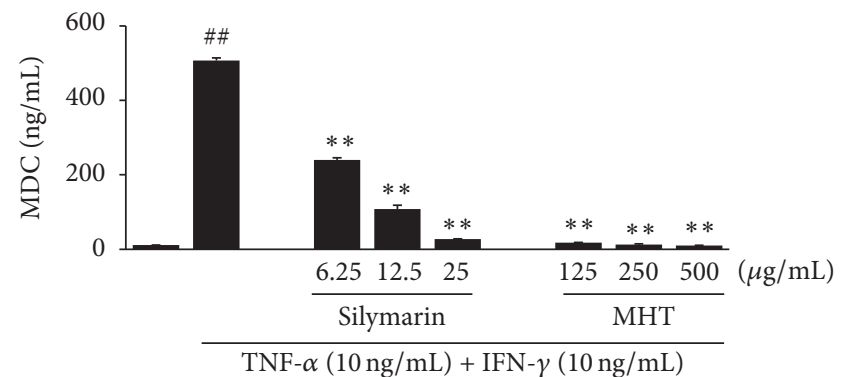

(b)



(d)

FIGURE 3: MHT inhibits production of inflammatory chemokines in TNF- $\alpha$ and IFN- $\gamma$-stimulated HaCaT cells. Production of TARC (a), MDC (b), RANTES (c), and IL-8 (d) were measured using the culture supernatants from cells treated with MHT (125, 250, or 500 $\mu \mathrm{g} / \mathrm{mL})$ and TNF- $\alpha$ and IFN- $\gamma$ (each $10 \mathrm{ng} / \mathrm{mL}$, TI) for $24 \mathrm{~h}$. Silymarin $(6.25,12.5$, or $25 \mu \mathrm{g} / \mathrm{mL}$ ) was used as positive control. Values were expressed as mean \pm SEM of three independent experiments. ${ }^{\# \#} P<0.01$ versus vehicle control cells; ${ }^{* * *} P<0.01$ versus TI-treated cells.

manner. We next analyzed the effects of MHT on chemokine mRNA expression in HaCaT cells costimulated with TI. Consistent with the results of ELISAs, stimulation with TI significantly increased the expression of TARC, MDC, RANTES, and IL-8 mRNA in HaCaT cells. Treatment of the cells with MHT significantly reduced the expression of TARC, MDC, RNATES, and IL-8 mRNA compared with TI in a dose-dependent manner (Figure 4 ). These results demonstrate that MHT can modulate production and expression of TI-stimulated Th2 inflammatory chemokine in $\mathrm{HaCaT}$ keratinocytes.

3.4. Effects of MHT on TNF- $\alpha$ and IFN- $\gamma$-Activated STAT1 Phosphorylation in $\mathrm{HaCaT}$ Keratinocytes. We investigated the effect of MHT on phosphorylation of STAT1 in keratinocytes. HaCaT cells were pretreated with MHT for $1 \mathrm{~h}$ and then stimulated with TI for $30 \mathrm{~min}$. MHT suppressed the TI-stimulated phosphorylation of STAT1 (Figure 5(a)). We also confirmed the effect of MHT on nuclear translocation of STAT1 by immunofluorescence analysis. STAT1 protein at cytosol was translocated to nucleus after TI stimulation whereas it was inhibited by MHT treatment (Figure 5(b)). These results indicate that MHT inhibits TI-stimulated STAT1 activation in HaCaT keratinocytes.

\section{Discussion}

MHT, which consists of six herbal medicines, is one of the traditional Korean medicine prescriptions and has been used for the treatment of headache, fever, and arthralgia which result from common cold in Korea [10]. The main ingredients of the MHT are known as follows: alkaloids (e.g., ephedrine and pseudoephedrine) from Ephedrae Herba [11], coumarin (e.g., coumarin, cinnamic acid, and cinnamaldehyde) from Cinnamomi Ramulus [12,13], triterpene saponins (e.g., glycyrrhizin) and flavonoids (e.g., liquiritin and liquiritigenin) from Glycyrrhizae Radix et Rhizoma [14], cyanoglucosides (e.g., amygdalin) from Armeniacae Semen [11], phenols (e.g., 6-gingerol and 6-shogaol) from Zingiberis Rhizoma Crudus [15], and phenylpropanoids (e.g., ferulic acid) and flavonoids (e.g., kaempferol) from Allii Radix [16]. Among the various ingredients, we try simultaneous determination of the nine components ephedrine $\mathrm{HCl}$, amygdalin, liquiritin apioside, liquiritin, coumarin, cinnamic acid, cinnamaldehyde, glycyrrhizin, and 6-gingerol in MHT water extract by a HPLC-PDA method. Consequently, amygdalin $(21.57 \mathrm{mg} / \mathrm{g})$, ephedrine $\mathrm{HCl}(13.80 \mathrm{mg} / \mathrm{g})$, and cinnamaldehyde $(12.52 \mathrm{mg} / \mathrm{g})$, marker compounds of Armeniacae Semen, Ephedrae Herba, and Cinnamomi Ramulus, respectively, were detected as major compounds.

Among the major compounds of MHT, amygdalin, ephedrine $\mathrm{HCl}$, and cinnamaldehyde have been reported to exhibit the effects of inflammatory responses through attenuation of iNOS, COX-2 expression via nuclear factorkappa B signaling pathway, and PI3K/Akt/GSK3 $\beta$ pathway [17-19]. However, the effects of MHT on skin inflammatory diseases have not been reported. In the present study, we found that MHT inhibited production and mRNA expression 




(a)



TNF- $\alpha(10 \mathrm{ng} / \mathrm{mL})+\mathrm{IFN}-\gamma(10 \mathrm{ng} / \mathrm{mL})$



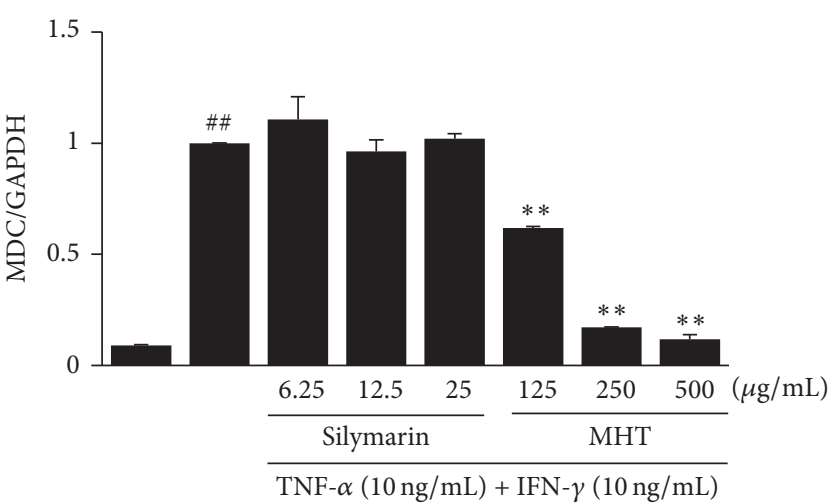

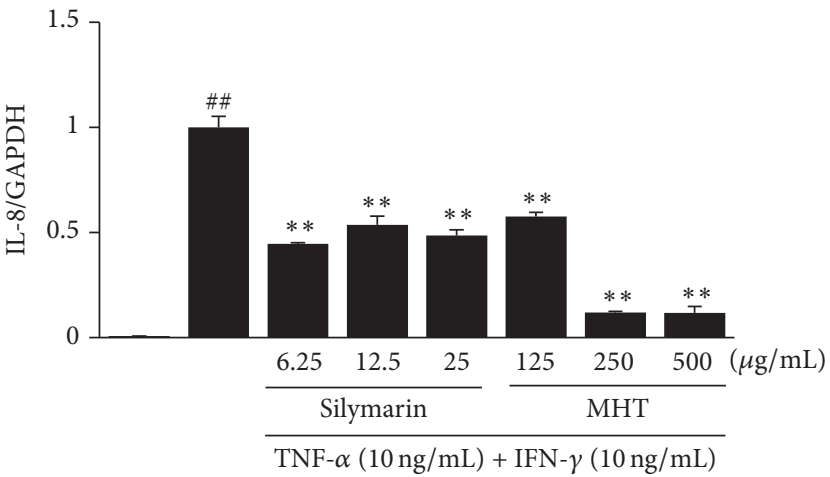

(b)

FIGURE 4: MHT inhibits the mRNA expression of inflammatory chemokines in TNF- $\alpha$ and IFN- $\gamma$-stimulated HaCaT cells. (a) RT-PCR was performed to determine the mRNA expression levels of TARC, MDC, RANTES, and IL-8. (b) The intensities of the PCR bands. Values were expressed as mean \pm SEM of three independent experiments. ${ }^{\# \#} P<0.01$ versus vehicle control cells; ${ }^{*, * *} P<0.01$ versus TI-treated cells.

of inflammatory chemokines such as TARC, MDC, RANTES, and IL- 8 by suppressing STAT1 activation in TI-stimulated keratinocytes.

Chemokine products play important roles in the development of inflammatory cells in the skin. The secretion of these inflammatory chemokines is the first recruitment of inflammatory skin diseases and can be observed in immune cells, including lymphocytes, keratinocytes, and mast cells, which are activated by various stimuli [20]. Keratinocytes may promote an amplification on the inflammatory response with the production of TNF- $\alpha$ and IFN- $\gamma$. Stimulated keratinocytes have been reported as important sources of proinflammatory chemokines, including TARC, MDC, RNATES, and IL-8, which affect T lymphocyte differentiation and the recruitment of leukocytes to skin inflammatory diseases such as $\mathrm{AD}[21,22]$. Consistent with the results of previous studies, we found that stimulation with TI markedly increased the production and mRNA expression of TARC, MDC, RNATES, and IL- 8 in HaCaT keratinocytes. By contrast, MHT treatment decreased both the production and mRNA expression of these chemokines compared with the TI-stimulated cells. These results indicate that MHT may have the inhibitory activity against skin inflammation through regulation of chemokines expression in keratinocytes.

STAT1 is a crucial molecule for the IFN- $\gamma /$ cytokine signaling pathways [22]. These activated pathways can modulate 




(a)

Control
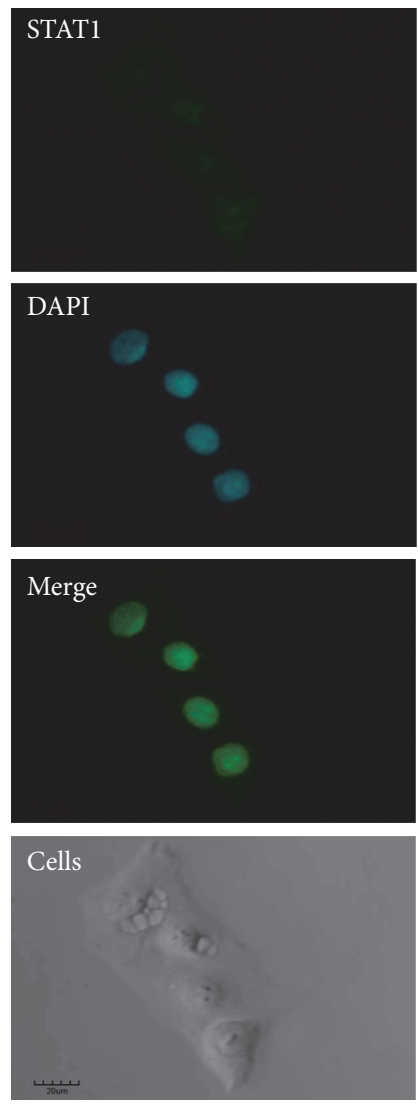

TNF- $\alpha+$ IFN- $\gamma(\mathrm{TI})$
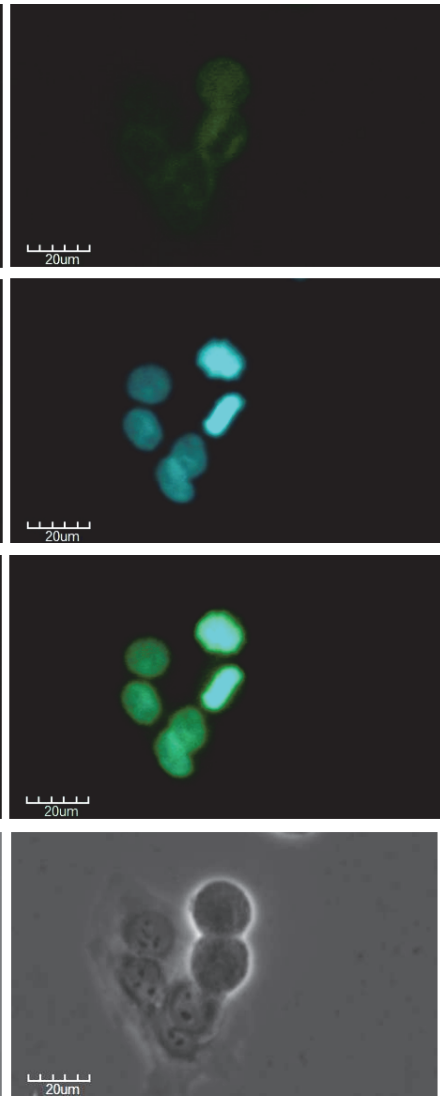

(b)
$\mathrm{TI}+\mathrm{MHT}$


FIGURE 5: MHT suppresses STAT1 activation in TNF- $\alpha$ and IFN- $\gamma$-stimulated HaCaT cells. (a) Phosphorylation of STAT1 was measured using cells cotreated with MHT $(125,250$, or $500 \mu \mathrm{g} / \mathrm{mL})$ and TNF- $\alpha$ and IFN- $\gamma$ (each $10 \mathrm{ng} / \mathrm{mL}$, TI) for 30 min by western blotting. (b) Cellular localization of STAT1 was analyzed by immunofluorescence staining. Cells were cotreated with MHT (500 $\mu \mathrm{g} / \mathrm{mL})$ and TI for $30 \mathrm{~min}$ on glass coverslips and incubated with anti-STAT1 and FITC-conjugated secondary antibodies. The cells were fixed in $4 \%$ (v/v) methanol-free formaldehyde solution ( $\mathrm{pH}$ 7.4), stained with anti-STAT1 (green). The stained cells were mounted in medium containing DAPI (blue) and visualized under an Olympus FLUOVIEW FV 10i confocal microscope.

the secretion of inflammatory mediators including TARC, MDC, and RANTES. Inhibition of STAT1 phosphorylation is considered to be an important step in treating skin inflammatory diseases [23]. To confirm the action mechanisms of the effect of MHT on the STAT1 activated by TI-stimulated $\mathrm{HaCaT}$ keratinocytes, we performed immunoblotting and immunocytochemistry assays (using anti-STAT1 antibody). In our study, MHT inhibited TI-stimulated STAT1 phosphorylation and nuclear translocation in $\mathrm{HaCaT}$ cells. These results suggest that MHT might block the induction inflammatory chemokines production by inhibiting STAT1 phosphorylation in TI-stimulated keratinocytes.

In summary, our present study demonstrates that MHT inhibited the production and mRNA expression of inflammatory chemokines including TARC, MDC, RANTES, and IL-8 by suppressing the activation of STAT1 in TI-stimulated $\mathrm{HaCaT}$ keratinocytes. Further investigation is required to elucidate the detailed mechanisms involved in anti-skin 
inflammatory diseases by MHT and to further confirm the inhibitory effect of MHT in skin diseases using an in vivo experimental model.

\section{Competing Interests}

The authors declare that they have no conflict of interests.

\section{Authors' Contributions}

Hye-Sun Lim, Hyeun-Kyoo Shin, and Soo-Jin Jeong conceived and designed the experiments; Hye-Sun Lim, ChangSeob Seo, Seong-Eun Jin, Sae-Rom Yoo, Mee-Young Lee, and Soo-Jin Jeong performed the experiments; Hye-Sun Lim and Soo-Jin Jeong analyzed the data; Hye-Sun Lim and Soo-Jin Jeong wrote the paper.

\section{Acknowledgments}

This work was supported by a Grant from the Korea Institute of Oriental Medicine (no. K16251).

\section{References}

[1] L. F. Eichenfield, C. N. Ellis, A. J. Mancini, A. S. Paller, and E. L. Simpson, "Atopic dermatitis: epidemiology and pathogenesis update," Seminars in Cutaneous Medicine and Surgery, vol. 31, no. 3, pp. S3-S5, 2012.

[2] J. H. Choi, S. W. Jin, B. H. Park et al., "Cultivated ginseng inhibits 2,4-dinitrochlorobenzene-induced atopic dermatitis-like skin lesions in NC/Nga mice and TNF- $\alpha / \mathrm{IFN}-\gamma$-induced TARC activation in HaCaT cells," Food and Chemical Toxicololgy, vol. 56, pp. 195-203, 2013.

[3] H. Saeki and K. Tamaki, "Thymus and activation regulated chemokine (TARC)/CCL17 and skin diseases," Journal of Dermatological Science, vol. 43, no. 2, pp. 75-84, 2006.

[4] H.-S. Lim, H. Ha, M.-Y. Lee et al., "Saussurea lappa alleviates inflammatory chemokine production in $\mathrm{HaCaT}$ cells and house dust mite-induced atopic-like dermatitis in Nc/Nga mice," Food and Chemical Toxicology, vol. 63, pp. 212-220, 2014.

[5] D.-J. Kwon, Y.-S. Bae, S. M. Ju et al., "Casuarinin suppresses TARC/CCL17 and MDC/CCL22 production via blockade of NF- $\kappa \mathrm{B}$ and STAT1 activation in HaCaT cells," Biochemical and Biophysical Research Communications, vol. 417, no. 4, pp. 12541259, 2012.

[6] X. Yang, W.-B. Peng, and X.-Q. Yue, "Syndrome differentiation and treatment of Taiyang disease in Shanghan Lun," Journal of Chinese Integrative Medicine, vol. 7, no. 2, pp. 171-174, 2009.

[7] T. Kubo and H. Nishimura, "Antipyretic effect of Mao-to, a Japanese herbal medicine, for treatment of type A influenza infection in children," Phytomedicine, vol. 14, no. 2-3, pp. 96101, 2007.

[8] Y. Toriumi, T. Kamei, K. Murata, I. Takahashi, N. Suzuki, and O. Mazda, "Utility of maoto in an influenza season where reduced effectiveness of oseltamivir was observed-a clinical, non-randomized study in children," Forschende Komplementarmedizin, vol. 19, no. 4, pp. 179-186, 2012.

[9] C.-H. Ma, Z.-Q. Ma, Q. Fu, and S.-P. Ma, "Ma Huang Tang ameliorates asthma though modulation of Th1/Th2 cytokines and inhibition of Th17 cells in ovalbumin-sensitized mice,"
Chinese Journal of Natural Medicines, vol. 12, no. 5, pp. 361-366, 2014.

[10] J. Hur, “Donguibogam," Seoul, Korea, p. 380, 666, 2004.

[11] Korean Pharmacopoeia Committee, The Korean Pharmacopoeia, Shinilbooks, Seoul, South Korea, 10th edition, 2013.

[12] K. Liang, S. Cui, Q. Zhang, K. Bi, Z. Qian, and Y. Jia, "UPLC simultaneous determination of five active components in Cinnamomi Ramulus," Zhongguo Zhongyao Zazhi, vol. 36, no. 23, pp. 3298-3301, 2011.

[13] K.-C. Wen, C.-Y. Huang, and F.-S. Liu, "Determination of cinnamic acid and paeoniflorin in traditional chinese medicinal preparations by high-performance liquid chromatography," Journal of Chromatography A, vol. 593, no. 1-2, pp. 191-199, 1992.

[14] Q. Zhang and M. Ye, "Chemical analysis of the Chinese herbal medicine Gan-Cao (licorice)," Journal of Chromatography A, vol. 1216, no. 11, pp. 1954-1969, 2009.

[15] H.-R. Lee, J.-H. Lee, C.-S. Park et al., "Physicochemical properties and antioxidant capacities of different parts of ginger (Zingiber officinale Roscoe)," Journal of the Korean Society of Food Science and Nutrition, vol. 43, no. 9, pp. 1369-1379, 2014.

[16] M. Parvu, A. Toiu, L. Vlase, and E. Alina Parvu, "Determination of some polyphenolic compounds from Allium species by HPLC-UV-MS," Natural Product Research, vol. 24, no. 14, pp. 1318-1324, 2010.

[17] J.-M. Yun, S.-B. Im, M.-K. Roh et al., "Prunus yedoensis bark inhibits lipopolysaccharide-induced inflammatory cytokine synthesis by $\mathrm{I} \kappa \mathrm{B} \alpha$ degradation and MAPK activation in macrophages," Journal of Medicinal Food, vol. 17, no. 4, pp. 407-413, 2014.

[18] Y. Zheng, Y. Yang, Y. Li et al., "Ephedrine hydrochloride inhibits PGN-induced inflammatory responses by promoting IL-10 production and decreasing proinflammatory cytokine secretion via the PI3K/Akt/GSK3 $\beta$ pathway," Cellular and Molecular Immunology, vol. 10, no. 4, pp. 330-337, 2013.

[19] Y. F. Chen, Y. W. Wang, W. S. Huang et al., "Transcinnamaldehyde, an essential oil in cinnamon powder, ameliorates cerebral ischemia-induced brain injury via inhibition of neuroinflammation through attenuation of iNOS, COX2 expression and $\mathrm{NF} \kappa-\mathrm{B}$ signaling pathway," NeuroMolecular Medicine, vol. 18, no. 3, pp. 322-333, 2016.

[20] J. N. W. N. Barker, C. E. M. Griffiths, B. J. Nickoloff, R. S. Mitra, V. M. Dixit, and B. J. Nickoloff, "Keratinocytes as initiators of inflammation," The Lancet, vol. 337, no. 8735, pp. 211-214, 1991.

[21] Y. Shimada, K. Takehara, and S. Sato, "Both Th2 and Th1 chemokines (TARC/CCL17, MDC/CCL22, and Mig/CXCL9) are elevated in sera from patients with atopic dermatitis," Journal of Dermatological Science, vol. 34, no. 3, pp. 201-208, 2004.

[22] J. Nakazato, M. Kishida, R. Kuroiwa, J. Fujiwara, M. Shimoda, and N. Shinomiya, "Serum levels of Th2 chemokines, CCL17, CCL22, and CCL27, were the important markers of severity in infantile atopic dermatitis," Pediatric Allergy and Immunology, vol. 19, no. 7, pp. 605-613, 2008.

[23] T. Kisseleva, S. Bhattacharya, J. Braunstein, and C. W. Schindler, "Signaling through the JAK/STAT pathway, recent advances and future challenges," Gene, vol. 285, no. 1-2, pp. 1-24, 2002. 




The Scientific World Journal
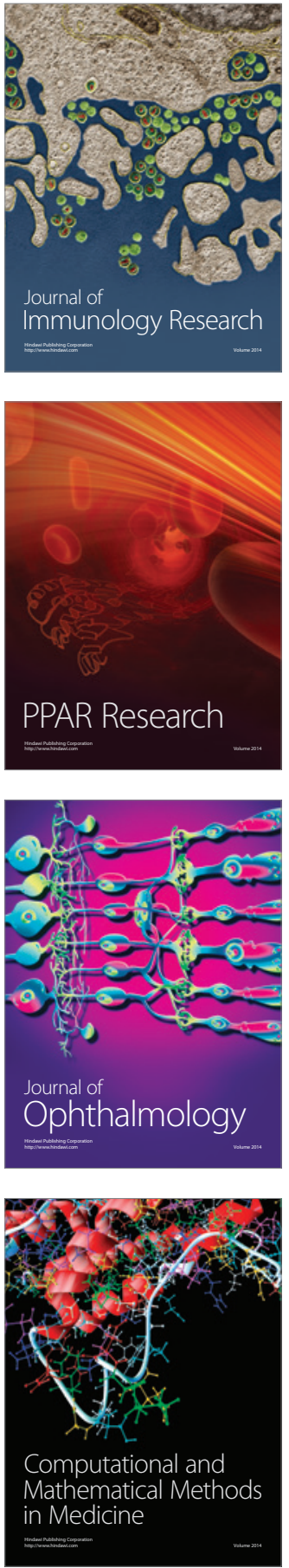

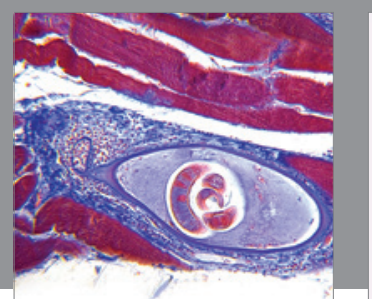

Gastroenterology Research and Practice



\section{Hindawi}

Submit your manuscripts at

http://www.hindawi.com

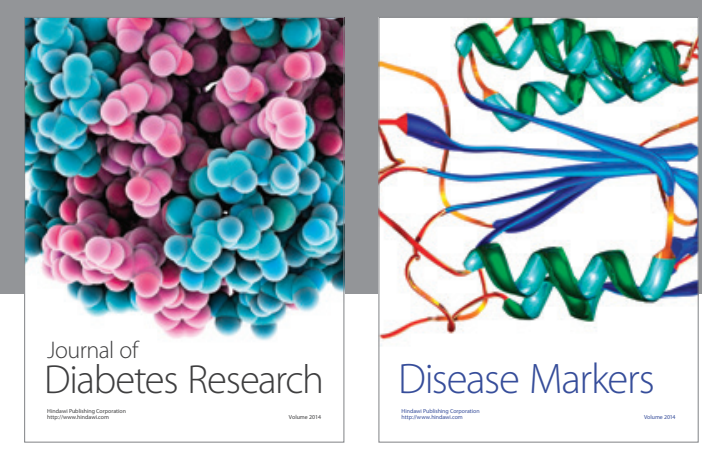

Disease Markers
